\title{
Non-invasively predicting differentiation of pancreatic cancer through comparative serum metabonomic profiling
}

\author{
Shi Wen ${ }^{1}$, Bohan Zhan², Jianghua Feng ${ }^{2^{*}}$, Weize Hu${ }^{1}$, Xianchao Lin ${ }^{1}$, Jianxi Bai ${ }^{1}$ and Heguang Huang ${ }^{1 *}$ (D)
}

\begin{abstract}
Background: The differentiation of pancreatic ductal adenocarcinoma (PDAC) could be associated with prognosis and may influence the choices of clinical management. No applicable methods could reliably predict the tumor differentiation preoperatively. Thus, the aim of this study was to compare the metabonomic profiling of pancreatic ductal adenocarcinoma with different differentiations and assess the feasibility of predicting tumor differentiations through metabonomic strategy based on nuclear magnetic resonance spectroscopy.

Methods: By implanting pancreatic cancer cell strains Panc-1, Bxpc-3 and SW1990 in nude mice in situ, we successfully established the orthotopic xenograft models of PDAC with different differentiations. The metabonomic profiling of serum from different PDAC was achieved and analyzed by using ${ }^{1} \mathrm{H}$ nuclear magnetic resonance (NMR) spectroscopy combined with the multivariate statistical analysis. Then, the differential metabolites acquired were used for enrichment analysis of metabolic pathways to get a deep insight.

Results: An obvious metabonomic difference was demonstrated between all groups and the pattern recognition models were established successfully. The higher concentrations of amino acids, glycolytic and glutaminolytic participators in SW1990 and choline-contain metabolites in Panc-1 relative to other PDAC cells were demonstrated, which may be served as potential indicators for tumor differentiation. The metabolic pathways and differential metabolites identified in current study may be associated with specific pathways such as serine-glycine-one-carbon and glutaminolytic pathways, which can regulate tumorous proliferation and epigenetic regulation.
\end{abstract}

Conclusion: The NMR-based metabonomic strategy may be served as a non-invasive detection method for predicting tumor differentiation preoperatively.

Keywords: Pancreatic ductal adenocarcinoma, Nuclear magnetic resonance, Metabonomics, Tumor differentiation

\section{Background}

Pancreatic ductal adenocarcinoma (PDAC) is one of the most malignant tumors with an extremely poor prognosis. Only about $7 \%$ of patients can be survived in 5 years, making PDAC the fourth leading cause of death among tumors [1]. Many risk factors have been correlated with prognosis, including tumor size [2, 3], lymph node metastasis $[3,4]$, nerve plexus invasion $[5,6]$, vascular invasion $[6,7]$, tumor differentiation $[2,3,8]$, surgical

\footnotetext{
*Correspondence: jianghua.feng@xmu.edu.cn; heguanghuang2@163.com; hhuang2@aliyun.com

${ }^{2}$ Department of Electronic Science, Fujian Provincial Key Laboratory of Plasma and Magnetic Resonance, Xiamen University, Xiamen 361005, China 'Department of General Surgery, Fujian Medical University Union Hospital, Fuzhou 350001, China
}

margin status $[3,9]$ and specific molecular prognostic factors $[10,11]$. Thereinto, poorly differentiated/high grade tumors are closely associated with poor outcome of the patients [12]. Furthermore, previous researches also linked tumor histological grading to an increased risk of early death within 1 year [13, 14]. As an important component of early mortality risk score, tumor differentiation can help to assessing short-term tumorrelated mortality $[14,15]$. Given the important role of tumor differentiation in PDAC management, increased interest in preoperative tumor differentiation assessment were emerged in order to identify high-risk patients, which can benefit the most from neoadjuvant treatment [13, 16-19], even over than upfront surgery $[20,21]$. 
Thus, notarizing differentiation of tumors preoperatively can provide constructive information for prognostic evaluation and management of PDAC [22].

Conventionally, the preoperative assessments of tumor differentiation were conducted by tissue histological observations derived from fine needle aspiration. This method has been realized to be an effective way to grade the pancreatic neuroendocrine tumors and intraductal papillary mucinous neoplasms [23, 24]. However, this technique is highly invasive for many patients and the achievable samples are too limited to give a reliable histological grading, making this technique still being far away from application in clinical PDAC differentiation assessment [19]. Thus, it would be of great importance to develop an easily acceptable and reliable method to assess the differentiation of PDAC preoperatively.

Nuclear magnetic resonance (NMR) spectroscopybased metabonomic technique is a promising diagnostic tool with the advantages of high sensitivity, non-invasion and high throughput. This technique can analyze the disease-related metabonomic differences occurred in various types of biosamples (etc. tissues, body fluids and cells) to identify differential metabolites and further biomarkers contributed to establishment of recognition models for diagnosis. At present, NMR-based diagnostic strategy has demonstrated a favorable clinical performance in many diseases [25-31]. Particularly noticeable, magnetic resonance spectroscopy have also been recommended for diagnosis of brain, prostate and breast cancer in European cancer conference [29]. In addition, by using NMR-based methods, many reports on detecting PDAC in vivo or in vitro have showed an encouraging result to distinguish PDAC from not only the normal but also other benign lesions [32-35]. Therefore, in present study, we used ${ }^{1} \mathrm{H}$ NMR spectroscopy to analyze serum metabonomes from PDAC mice models established by implantations of Panc-1, BxPC-3 and SW1990 (being poor, poor to moderate and moderate to well differentiated [36-39], respectively) cell strains on pancreas, thus, to assess the feasibility of this strategy in predicting the differentiation of tumor.

\section{Methods}

\section{Cell culture and animals feeding}

PDAC cell strains (Panc-1, BxPC-3 and SW1990, Catalog NO. SCSP-535, TCHu 12 and TCHu 201) were obtained from Shanghai Institute of Cell Biology, Chinese Academy of Sciences (Shanghai, China) authenticated with short tandem repeat test and mycoplasma culture. At the circumstance of $5 \% \mathrm{CO}_{2}$ and $37{ }^{\circ} \mathrm{C}$, these strains were incubated in dulbecco's modified eagle medium (DMEM, Gibco, Thermo Fisher Scientific Inc., USA) added with $10 \%$ fetal bovine serum (Gibco) in cell incubator (3110, Thermo Scientific). Then, cells were digested by $0.125 \%$ trypsinogen (Life Technologies, Grand Island, NY, USA) for the passage with the ratio of 1:2-4 every 2-3 days. BALB/c nude mice (male, 4 weeks, weighing 18-20 g), purchased from Shanghai Slac laboratory animals Co., Ltd. (NO: SCXK (HU) 2012-0002), were bred in Fujian Medical University Animals Centre (Fuzhou, china) with a standard SPF-grade laboratory conditions.

\section{Establishment of animal models}

This experimental protocol was in accordance with the principles of National Institutes of Health guide for the care and use of laboratory animals and approved by Ethical Committee of Fujian Medical University. Three PDAC cell strains in the exponential phase were digested with $0.125 \%$ trypsinogen, washed by phosphate buffered saline (PBS) for three times, then collected and resuspended in PBS $\left(1 \times 10^{7}\right.$ cells per milliliter). After skin degerming, the cell suspension liquids were subcutaneously injected into the axilla of mice (one cell strain each mouse), followed by a month of normal feeding. The tumors with a size of 5 to $10 \mathrm{~mm}$ in diameter generated in the injected positions of mice. Consequently, the mice were executed by a mercy killing, and the tumor tissues of Panc-1, BxPC-3 and SW1990 were carefully collected and divided into pieces of $1 \mathrm{~mm}^{3}$ for implantation in situ.

Forty-five mice were randomly divided into 3 groups using random number table. Before surgery, all mice have a 12-h fasting without drink-deprivation. A 2-cm horizontal incision was made on the middle of abdominal wall to expose the pancreas. One piece of tumors was placed on the body or tail of pancreas and fixed with biogum (BaiYun medical glue Co., Ltd., Guangzhou, China), followed by carefully organ restoration and suture. Three groups were dealt with tumor tissues of Panc-1, BxPC-3 and SW1990, respectively ( $n=15$ for each).

\section{Tissues samples collection and preparation}

Thirty days after surgeries, $1 \mathrm{~mL}$ of blood from each group was collected by aortic puncture under continuous airway anesthesia of isoflurane (Jiupai pharmaceutical Co., Ltd., Shijiazhuang, China) and stored in clear 1.5-mL Eppendorf tubes. After standing for $30 \mathrm{~min}$, the blood went through a $10-\mathrm{min}$ centrifugation at $10,000 \mathrm{~g}$ and $4{ }^{\circ} \mathrm{C}$. The supernate was collected and immediately frozen by liquid nitrogen and stored at $-80{ }^{\circ} \mathrm{C}$. For the detection of ${ }^{1} \mathrm{H}$ NMR spectroscopy, $400 \mu \mathrm{L}$ of serum were melted on the surface of ice, and then mixed with $200 \mu \mathrm{L}$ of $90 \mathrm{mM}$ deuterated phosphate buffer $\left(\mathrm{NaH}_{2} \mathrm{PO}_{4}\right.$ and $\mathrm{K}_{2} \mathrm{HPO}_{4}, \mathrm{pH}$ 7.4). The mixture of serum and buffer were centrifuged again, and finally, $550 \mu \mathrm{L}$ of the supernate was moved into $5-\mathrm{mm}$ NMR tubes (ST500, NORELL, Inc., Morganton, North Carolina, USA). 


\section{Detection of 1H NMR spectroscopy and preprocessing} The ${ }^{1} \mathrm{H}$ NMR spectroscopy of serum samples were performed on a Varian NMR system (Agilent Technologies Co, Palo Alto, California, USA) with a $500.13 \mathrm{MHz}$ of proton frequency at the temperature of 298 K. For each sample, a water-suppressed CPMG (Carr-Purcell-Meiboom-Gill) spin-echo pulse sequence $\left(\mathrm{RD}-90^{\circ}-\left(\mathrm{\tau}-180^{\circ}-\mathrm{\tau}\right)_{\mathrm{n}}-\mathrm{ACQ}\right)$ was used to acquire the NMR spectrum. Herein, a total of 64 scans with a spectral width of $6 \mathrm{KHz}$ and a data point of $12 \mathrm{~K}$ were accumulated for all spectra. Spin-echo loop time $(2 \mathrm{n \tau})$ of $70 \mathrm{~ms}$ was applied with a relaxation delay of 2.0 s. The NMR spectra were processed by using MestReNova (V9.0.1, Mestrelab Research S. L., Spain). In order to increase the signal-to-noise ratio, all free induction decays were multiplied by an exponential weighting function equivalent to a $1 \mathrm{~Hz}$ linebroadening and subsequently disposed with Fourier transformation. To make the spectra more comparable, the manual phase rectifications and baseline corrections were conducted by using MestReNova. The chemical shifts were referenced to the double-peak of endogenic lactate at $\delta 1.33$ for metabolites identification. Automatically, the spectral regions $89.0-0.5$ of the processed NMR spectra were segmented into scatter integral regions of $0.002 \mathrm{ppm}$ with a removal of spectral region $\delta 6.40-5.50$ and $\delta 5.19-4.36$ to eliminate the impacts of residual water signal and urea signal, respectively. Finally, for each spectrum, the integrated data were normalized to the total sum of the spectrum in favour of multivariate statistical analysis.

\section{Multivariate statistical analysis}

The multivariate statistical analysis, including principal component analysis (PCA), partial least squares discriminant analysis (PLS-DA) and orthogonal partial least squares discriminant analysis (OPLS-DA), were performed in SIMCA-P ${ }^{+}$(V14.0 Umetrics, Sweden) to analyze the metabonomic differences between three PDAC groups. PCA, performed in the approach of mean-centered scaling, could simplify the normalized date into several components, which can roughly evaluate the clusters distributions and identify the existence of outlines. PLS-DA and OPLS-DA, which can be classified as supervised multivariate statistical analysis, were conducted in the approach of parato-scaling approach for better extraction and maximization of the metabonomic differences between PDAC groups. Furthermore, the OPLS-DA models coefficients, which were back-calculated from the coefficients, incorporated with the weight of the variables, and then to be plotted with color-coded coefficients to enhance interpretability of the models. As a result, the metabolites responsible for the metabonomic differences between groups can be extracted from the corresponding colorcoded loading plots and displayed visually. By assistance of MATLAB (V7.1, the Mathworks Inc., Natick, USA), the color-coded coefficient loading plots were drew and color-coded according to the absolute value of coefficient. That meant, in the loading plots, a warm-toned color (i.e. red) represents for the metabolites being positive or negative significant in distinguishing different groups while a cool-toned

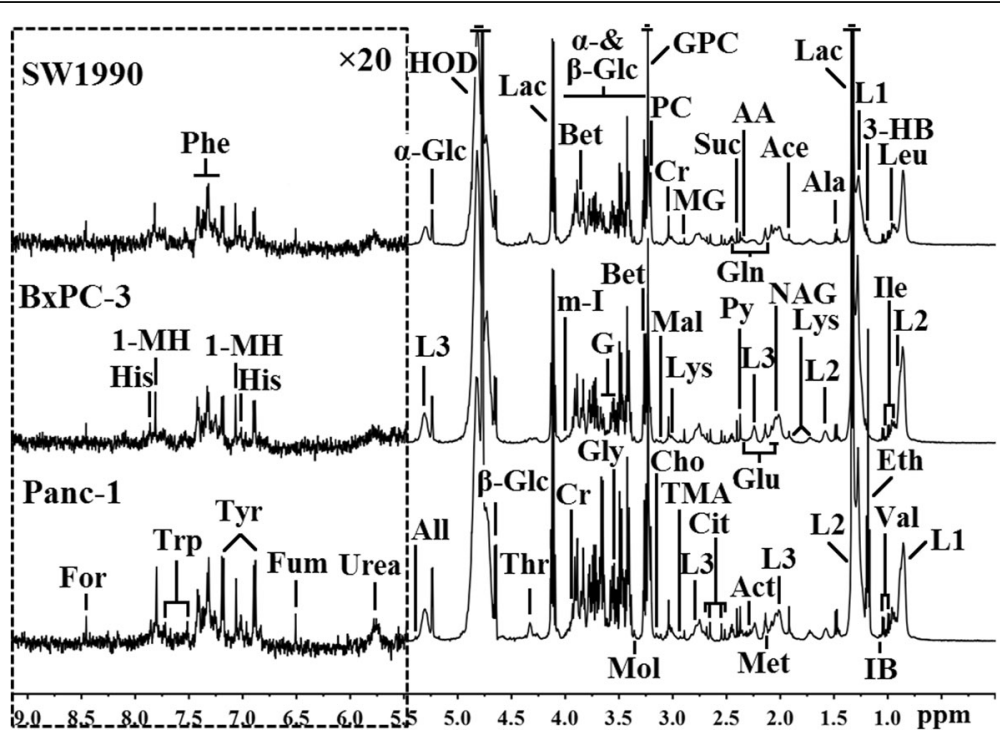

Fig. 1 Representative $500 \mathrm{MHz}{ }^{1} \mathrm{H}$ CPMG NMR spectra of serum samples from pancreatic cancer mice induced by the different differentiated cells. The spectral regions of $85.5-9.0$ (in the dashed box) were magnified 20 times compared with the regions of $80.0-5.5$ for the purpose of clarity. The abbreviations for peak assignments were noted in Table 1 
Table 1 The metabolites assignments from NMR spectra of serum from PDAC mice ${ }^{a}$

\begin{tabular}{|c|c|c|}
\hline Abbreviation & Metabolites & ${ }^{1} \mathrm{H}$ chemical shift(multiplicity) ${ }^{b}$ \\
\hline $1-\mathrm{MH}$ & 1-Methylhistidine & $7.06(\mathrm{~s}), 7.78(\mathrm{~s})$ \\
\hline $3-\mathrm{HB}$ & 3-Hydroxybutyrate & $1.20(\mathrm{~d}), 2.31(\mathrm{dd}), 2.40(\mathrm{~m}), 4.16(\mathrm{~m})$ \\
\hline Ace & Acetate & $1.92(\mathrm{~s})$ \\
\hline AA & Acetoacetate & $2.28(\mathrm{~s})$ \\
\hline Act & Acetone & $2.24(s)$ \\
\hline Ala & Alanine & $1.48(d)$ \\
\hline All & Allantoin & $5.39(s)$ \\
\hline Bet & Betaine & $3.27(\mathrm{~s}), 3.90(\mathrm{~s})$ \\
\hline Cho & Choline & $3.20(s)$ \\
\hline Cit & Citrate & $2.53(d), 2.67(d)$ \\
\hline $\mathrm{Cr}$ & Creatine & $3.04(\mathrm{~s}), 3.93(\mathrm{~s})$ \\
\hline Eth & Ethanol & $1.18(\mathrm{t}), 3.61(\mathrm{q})$ \\
\hline For & Formate & $8.46(s)$ \\
\hline Fum & Fumarate & $6.52(s)$ \\
\hline Glu & Glutamate & $2.08(\mathrm{~m}), 2.11(\mathrm{~m}), 2.35(\mathrm{~m}), 3.75(\mathrm{t})$ \\
\hline Gln & Glutamine & $2.14(\mathrm{~m}), 2.45(\mathrm{~m}), 3.75(\mathrm{t})$ \\
\hline G & Glycerol & $3.55(\mathrm{~m}), 3.66(\mathrm{dd}), 3.78(\mathrm{~m})$ \\
\hline GPC & Glycerolphosphocholine & $3.23(\mathrm{~s}), 4.33(\mathrm{~m})$ \\
\hline Gly & Glycine & $3.56(\mathrm{~s})$ \\
\hline His & Histidine & $7.08(s), 7.82(s)$ \\
\hline $\mathrm{HOD}$ & Residual water signal & $4.76(\mathrm{br})$ \\
\hline IB & Isobutyrate & $1.07(d)$ \\
\hline Ile & Isoleucine & $0.94(\mathrm{t}), 1.01(\mathrm{~d})$ \\
\hline L1 & LDL & $0.86(\mathrm{br}), 1.28(\mathrm{br})$ \\
\hline L2 & VLDL & $0.89(\mathrm{br}), 1.30(\mathrm{br}), 1.58(\mathrm{br})$ \\
\hline L3 & Unsaturated fatty acid & $2.04(\mathrm{br}), 2.24(\mathrm{br}), 2.76(\mathrm{br}), 5.31(\mathrm{br})$ \\
\hline Lac & Lactate & $1.33(\mathrm{~d}), 4.11(\mathrm{q})$ \\
\hline Leu & Leucine & $0.96(d)$ \\
\hline Lys & Lysine & $\begin{array}{l}1.46(\mathrm{~m}), 1.73(\mathrm{~m}), 1.91(\mathrm{~m}) \\
3.03(\mathrm{~m}), 3.76(\mathrm{t})\end{array}$ \\
\hline Mal & Malonate & $3.11(\mathrm{~s})$ \\
\hline Met & Methionine & $2.14(\mathrm{~s}), 2.63(\mathrm{t})$ \\
\hline MG & Methylguanidine & $2.83(\mathrm{~s}), 3.36(\mathrm{~s})$ \\
\hline Mol & Methanol & $3.36(s)$ \\
\hline$m-1$ & myo-Inositol & $3.52(\mathrm{dd}), 3.61(\mathrm{dd}), 4.07(\mathrm{~m})$ \\
\hline NAG & $\mathrm{N}$-acetyl glycoprotein & $2.03(s)$ \\
\hline Phe & Phenylalanine & $7.32(\mathrm{~d}), 7.37(\mathrm{t}), 7.42(\mathrm{dd})$ \\
\hline PC & Phosphocholine & $3.21(\mathrm{~s})$ \\
\hline Py & Pyruvate & $2.37(s)$ \\
\hline Suc & Succinate & $2.40(s)$ \\
\hline Thr & Threonine & $1.33(\mathrm{~d}), 4.26(\mathrm{~m})$ \\
\hline TMA & Trimethylamine & $2.89(\mathrm{~s})$ \\
\hline Trp & Tryptophan & $7.27(\mathrm{~m}), 7.30(\mathrm{~s}), 7.54(\mathrm{~d}), 7.73(\mathrm{~d})$ \\
\hline Tyr & Tyrosine & $6.90(d), 7.19(d)$ \\
\hline
\end{tabular}

Table 1 The metabolites assignments from NMR spectra of serum from PDAC mice ${ }^{a}$ (Continued)

\begin{tabular}{lll}
\hline Abbreviation & Metabolites & ${ }^{1} \mathrm{H}$ chemical shift(multiplicity) ${ }^{\mathrm{b}}$ \\
\hline Urea & Urea & $5.80(\mathrm{br})$ \\
Val & Valine & $0.99(\mathrm{~d}), 1.04(\mathrm{~d})$ \\
a-Glc & a-Glucose & $3.42(\mathrm{t}), 3.54(\mathrm{dd}), 3.71(\mathrm{t})$, \\
& & $3.73(\mathrm{~m}), 3.84(\mathrm{~m}), 5.24(\mathrm{~d})$ \\
$\beta$-Glc & $\beta$-Glucose & $3.24(\mathrm{ddb}), 3.41(\mathrm{t}), 3.46(\mathrm{~m})$, \\
& & $3.49(\mathrm{t}), 3.90(\mathrm{dd}), 4.65(\mathrm{~d})$ \\
\hline
\end{tabular}

${ }^{a} P D A C$ pancreatic ductal adenocarcinoma

${ }^{b}$ multiplicity:s, singlet; d, doublet; t, triplet; q, quartet; dd, doublets; $\mathrm{m}$, multiplet; br, broad resonance

color (i.e. blue) corresponds to the metabolites not being significant in discriminations. Moreover, to screen out differential metabolites, the cutoff value of correlation coefficients $(|\mathrm{r}|>0.576)$ was determined according to the statistical significance of the Pearson correlation coefficient test at the level of $P<0.05$ and $d f$ (degree of freedom) $=10$. In order to assess the quality and validity of models, the 10-fold cross validation and response permutation testing $(n=200)$ were performed and the corresponding parameters $R^{2}$ and $Q^{2}$ in the permutated plots presented the degree of model fitting and the potentially predictive ability of models, respectively.

\section{The metabolic pathways and interactions analysis}

The differential metabolites derived from multivariate statistical analysis were further analyzed for the metabolic pathways by using KEGG (www.genome.jp/kegg) and MBROLE 1.0 (http://csbg.cnb.csic.es/mbrole/) [40, 41].

\section{Results}

NMR spectral profiles of serum samples from Panc-1, BxPC-3, SW1990 groups

After visual confirmation for tumorgenesis, 12, 13, and 11 serum samples from Panc-1, BxPC-3 and SW1990 groups were included for the detections with ${ }^{1} \mathrm{H}$ NMR spectroscopy, respectively. Typical one-dimensional $500-\mathrm{MHz}{ }^{1} \mathrm{H}$ NMR spectra of serum samples from models induced by the different differentiated PDAC cells are presented in Fig. 1, which provided an integrated overview of all metabolites. Forty-seven metabolites were identified from the NMR spectra (Table 1) based on the relative literatures and public databases $[42,43]$. A certain degree of metabolic differences could be noticed between different PDAC groups visually such as ethanol and phosphocholine. But considering the high similarity of spectra, the metabonomic information acquired was quite limited and the multivariate statistic analysis will help to extract the detailed information. 

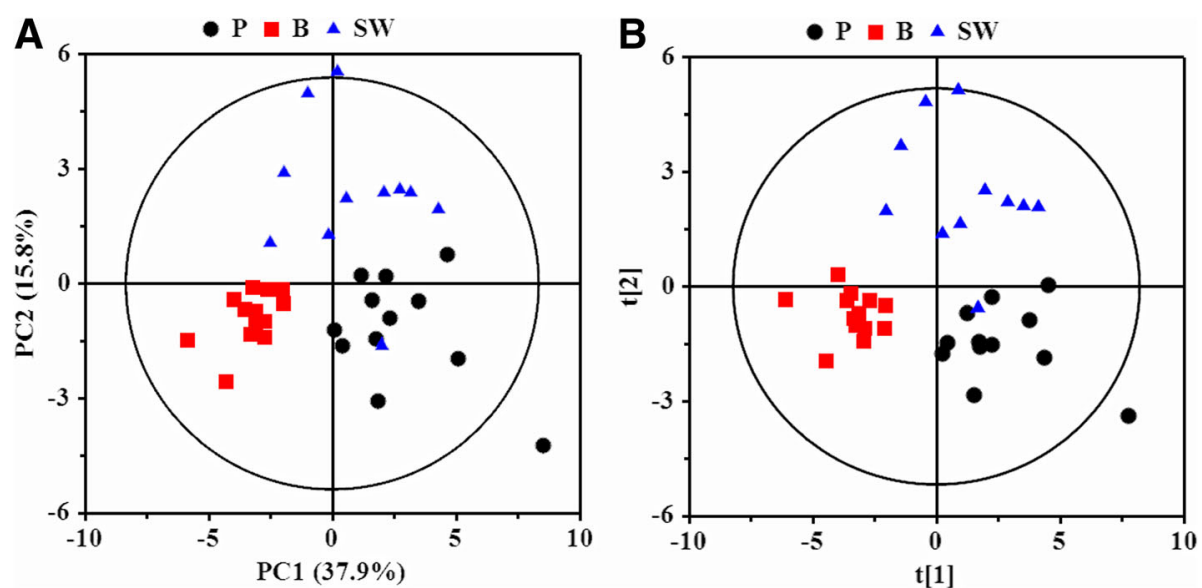

Fig. 2 The PCA (a) and PLS-DA (b) scores plots based on ${ }^{1} H$ NMR data of serums from PDAC groups. P, Panc-1; B, BXPC-3; SW, SW1990

Metabonomic characteristics of serum from the PDAC groups To show an overview of ${ }^{1} \mathrm{H}$ NMR data collected from the serum of Panc-1, BxPC-3, and SW1990 groups, the PCA and PLS-DA were performed. The PCA scores plot showed a certain degree of separated trends between the three PDAC groups (Fig. 2a) though a little overlap or dispersity was demonstrated, indicating their obvious metabonomic differences. In further, a greater discrimination in cluster distributions of Panc-1, Bxpc-3 and SW1990 could be observed visually in PLS-DA scores plot (Fig. 2b), demonstrating a significant differences with each other.

To get deep insight into the metabolites responsible for the metabonomic alterations occurred in three PDAC groups, pair-wise comparisons were conducted by using the PLS-DA combined with orthogonal projection (OPLSDA). The pronounced separations were demonstrated in OPLS-DA scores plots (Fig. 3 upper left panels) and the metabolites corresponding to the metabolic difference were marked in loading plots (Fig. 3 bottom panels). The summarized dominant metabolites, based on the cutoff value of correlation coefficient $(|\mathrm{r}|>0.576)$, and the correlation coefficients were listed in detail based on their biochemical types (Table 2). Overall, the levels of metabolites belonged to glycolysis and glutaminolysis, alcohols and amino acids were lower in SW1990 group while the high concentrations of choline and its derivatives were noticeable in Panc-1 group. The favorable fit and prediction parameters $\left(\mathrm{R}^{2}\right.$ and $\left.\mathrm{Q}^{2}\right)$ of the OPLS-DA models and the corresponding permutation test and probability ( $p$-value) via CV-ANOVA also confirmed the strong predictive ability of the models to guarantee a reliable identification of characteristic metabolites.

The biochemical pathways related with the metabonomic difference between PDAC groups

For better understanding of the bioinformation contained in discriminatory metabolites, the biochemical pathways were identified based on the differential metabolites derived from OPLS-DA of pair-comparisons and those with $p$-value less than 0.01 were demonstrated on Fig. 4. The $p$-value for pathway identification were calculated automatically by the MBROLE [40].

In the analysis to compare SW1990 with Bxpc-3, the numerous amino acid-related pathways were noticeable, including metabolism of essential and non-essential amino acids, the biosynthesis of aminoacyl-tRNA and $\mathrm{ABC}$ transporters. In addition, the pathways related with glycolysis involving pyruvate, galactose, glutamine and glutamate were also identified as differential features to distinguish the Bxpc-3 from the SW1990. Meanwhile, except the pathways of lysine, histidine and thiamine metabolisms, most pathways involved in Bxpc-3 vs SW1990 were also identified in the comparison between Panc-1 and Sw1990. In addition, the pathways of glycerophospholipid metabolism and the degradation of valine, leucine and isoleucine were also identified to be a signature contributed to distinguish Panc-1 from SW1990. In term of metabolic diversity between Panc-1 and BxPC-3, the metabolic discrimination seems to be quite limited where only a few pathways related with amino acids and glycerophospholipid metabolism were identified.

\section{Discussion}

In this study, we tried to evaluate the potential value of non-targeted NMR strategy to predict the tumor differentiation. Since many factors (e.g., drugs, operations) could influence the metabonomic characteristics of serum from patients. We chose three PDAC strains, Panc-1, BxPC-3 and SW1990 which can form tumors in vivo with typical histopathologic characters from poor, poor to moderate and moderate to well differentiation respectively [36-39] to establish PDAC models for research. By using animal models, the interference factors can be furthest eliminated. It is beneficial to purify 
A
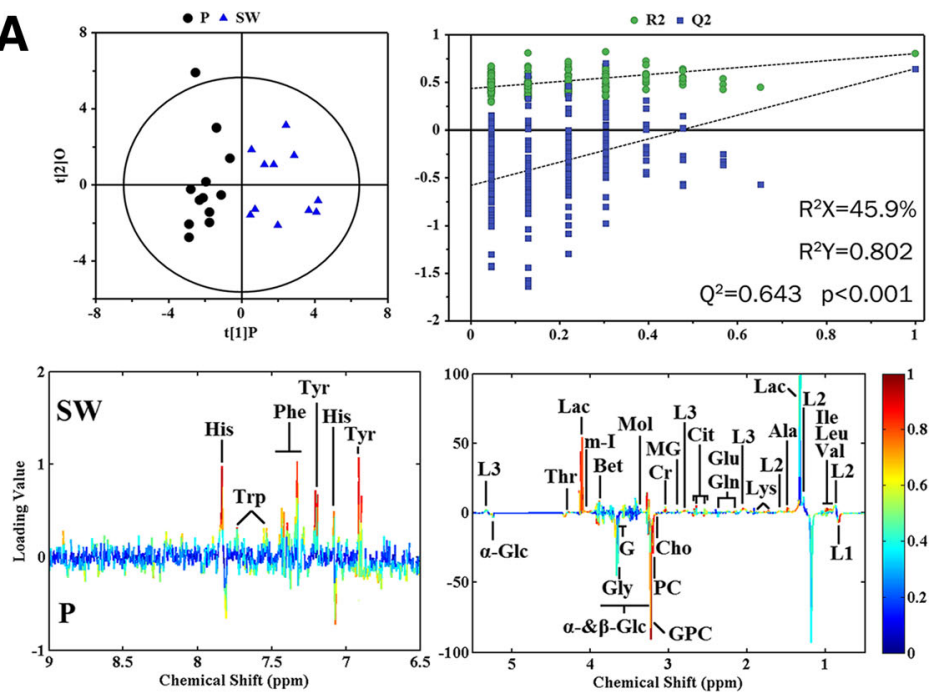

B
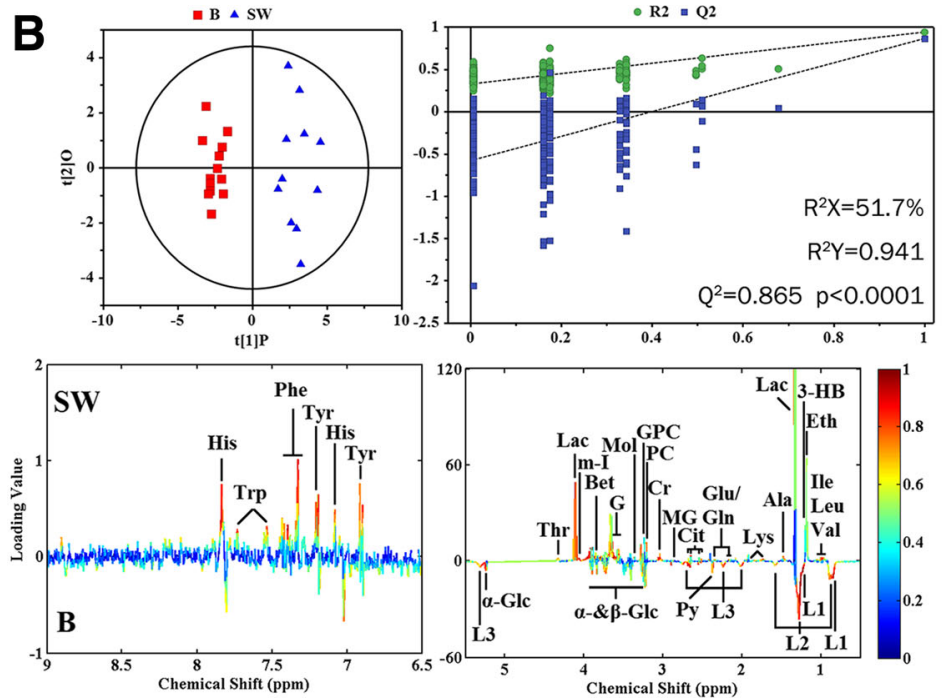

C
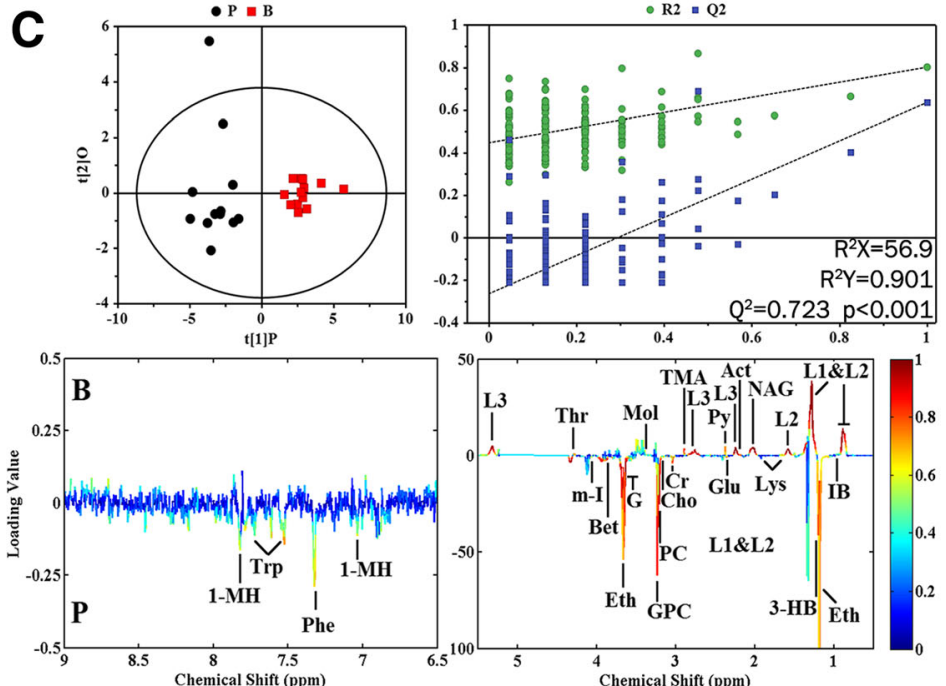

Fig. 3 (See legend on next page.) 
(See figure on previous page.)

Fig. 3 OPLS-DA scores plots (upper left panels) and plots of permutation tests $(n=200)$ (upper right panels) derived from ${ }^{1} H$ NMR spectra of serum samples and corresponding coefficient loading plots (bottom panels) from the pair-wise comparisons between Panc-1, Bxpc-3 and SW1990 groups. a. Panc-1 vs SW1990, b. BxPC-3 vs SW1990, c. Panc-1 vs BxPC-3. The color map shows the significance of metabolites variations between the two classes. Keys of the assignments were shown in Table 1. P, Panc-1; B, BxPC-3; SW, SW1990

serum metabonomic alteration caused by tumor with different differentiation and also specify the association between tumor differentiation and serum metabonomes. To amplify the metabolic difference between the tumors in different differentiations, all groups were compared directly. Given most of clinical patients were diagnosed with moderately differentiated PDAC and the significant clinical value for the identification of tumors in poor differentiation, we focus on the metabonomic difference between SW1990 and other two strains.

\section{Comparative low levels of lactate, glutamate and glutamine indicate a poor differentiation}

In present study, we found that the high concentration of citrate, lactate, glutamate and glutamine can help to distinguish the SW1990 from Panc-1 and Bxpc-3. Being well known, the tumor metabolic reprogramming has been validated to be the cornerstone for malignant transformation and one common composition in this process is the aerobic glycolysis (Warburg effect). Through the aerobic glycolysis, rather than tricarboxylic acid (TCA) cycle, the tumor cells derive the predominant ATP/energy and generate extensive lactate from pyruvate to result in environmental acidosis which promote the spreading of the tumor cells [44]. Meanwhile, the lactate generated from hypoxic PDAC can be taken up by normoxic PDAC cells nearby as fuel to maintain proliferation, creating a phenomenon called tumor symbiosis [45]. Thus, the tumor metabolic impact upon the level of lactate in peripheral circulation may be determined by the dynamic balance of release and uptake of lactate around tumor microenvironment. Our outcome indicates that the tumor with a poorer differentiation could induce a lower concentration of lactate in serum relative to that with a better differentiation, which may be due to a stronger ability of lactate recirculation. It's also implied by inconsistent variation trends of lactate in serum reported by previous studies [46, 47]. In addition, due to the breakdown of TCA cycle, glutaminolysis is enhanced in PDAC cells to generate TCA intermediates (e.g. malate, oxaloacetate and citrate) which is called anaplerosis reaction, and subsequently served as building blocks for synthesis of lipid and non-essential amino acids [48]. Besides, glutamine can also act as fuel to support energy metabolism through aspartate, oxaloacetate and pyruvate transformation process, thus promoting growth of pancreatic cancer via Kras-regulated metabolic pathway [49]. Therefore, the significantly low levels of glutamine, glutamate and citrate may indicate that the tumor with poorer differentiation may provide a more dramatic glutaminolysis and deprive more glutamine and glutamate from peripheral circulation.

\section{Comparative low levels of amino acids in serum imply poor differentiation}

Likewise, the higher concentrations of amino acids could also contribute to the distinguishing of the SW1990 from Panc-1 and Bxpc-3, which could serve as key participants in the cancer metabolism reprogramming. Under the influence of the abnormal expression of oncogenes and tumor suppress genes, the anabolic metabolism and transport of amino acid were tremendously enhanced for rapid proliferation of cancer cells. To provide required nutrients for cancer growth, the catabolic metabolism of whole-body tissue would be enhanced, leading to an increased circulating amino acids at the early stage of PDAC [50]. But the catabolic metabolism cannot maintain in a high level for a long time and end in a severe nutritional imbalance called cachexia, thus creating a decrease of amino acids in serum at last. In this process, L-type amino-acid transporter 1 (LAT-1), the most important transporter of neutral amino acids, plays a key role in internalized transportation of essential amino acids (EAAs) in PDAC. As previous reports demonstrated, the overexpression of LAT-1 can promote cancer growth via mammalian target-of-rapamycin (mTOR) and serve as a prognostic factor in PDAC $[51,52]$. Thus, the higher concentration of EAAs in SW1990 group than in Panc-1 and BxPC-3 group indicates that the tumors with poor differentiation may have a higher expression of LAT1 and nutritional stress from rapid proliferation, which can associated with poor prognosis.

With regard to the non-essential amino acids (NEAAs), several pathways were involved to enhance their biosynthesis and utilization for cell proliferation. As noted above, the accumulated glycolysis intermediates could also promote the biosynthesis of glycine, serine and threonine through 3-phospho-D-glycerate pathway. In addition, the increased glutaminolysis provides numerous substrates (e.g. isocitrate, malate, alphaketoglutaric acid) not only to supply the lipids synthesis but also to promote the biosynthesis of alanine and aspartate. Besides being used as building blocks and fuels for cell proliferation, NEAAs have been indicated to bridge the interplay metabolism and epigenetics, thus 
Table 2 OPLS-DA coefficients of metabolites in different pair-comparisons derived from NMR-data

\begin{tabular}{|c|c|c|c|}
\hline \multirow[t]{2}{*}{ Metabolites } & \multicolumn{3}{|l|}{$r^{a}$} \\
\hline & $\begin{array}{l}\text { BXPC-3 vs } \\
\text { SW1990 }\end{array}$ & $\begin{array}{l}\text { Panc-1 Vs } \\
\text { SW1990 }\end{array}$ & $\begin{array}{l}\text { Panc-1 vs } \\
\text { BxPC-3 }\end{array}$ \\
\hline \multicolumn{4}{|l|}{ Glycolysis and glutaminolysis } \\
\hline a-Glucose & -0.788 & -0.631 & - \\
\hline$\beta$-Glucose & -0.735 & -0.842 & - \\
\hline Citrate & 0.817 & 0.921 & - \\
\hline Glutamate & 0.808 & 0.747 & -0.789 \\
\hline Glutamine & 0.767 & 0.856 & - \\
\hline Lactate & 0.906 & 0.905 & - \\
\hline pyruvate & -0.880 & - & 0.793 \\
\hline Succinate & - & - & - \\
\hline \multicolumn{4}{|l|}{ Carboxylic acids and derivatives } \\
\hline Acetate & - & - & - \\
\hline Formate & - & - & - \\
\hline Fumarate & - & - & - \\
\hline Isobutyrate & - & - & -0.709 \\
\hline Malonate & - & - & -0.648 \\
\hline \multicolumn{4}{|l|}{ Alcohols } \\
\hline Ethanol & 0.879 & - & -0.804 \\
\hline Methanol & 0.702 & 0.760 & 0.667 \\
\hline myo-Inositol & 0.889 & 0.817 & -0.877 \\
\hline Glycerol & 0.935 & 0.784 & -0.916 \\
\hline \multicolumn{4}{|l|}{ Lipid } \\
\hline LDL & -0.899 & -0.847 & 0.912 \\
\hline VLDL & -0.774 & 0.720 & 0.921 \\
\hline Unsaturated fatty acid & -0.899 & -0.847 & 0.912 \\
\hline \multicolumn{4}{|l|}{ ketone body } \\
\hline 3-Hydroxybutyrate & 0.747 & - & -0.636 \\
\hline Acetoacetate & - & - & - \\
\hline Acetone & -0.760 & - & 0.912 \\
\hline \multicolumn{4}{|l|}{ Choline and derivatives } \\
\hline Choline & - & -0.836 & -0.841 \\
\hline Glycerolphosphocholine & 0.671 & -0.912 & -0.894 \\
\hline Phosphocholine & 0.736 & -0.832 & -0.892 \\
\hline \multicolumn{4}{|l|}{ Amino acid } \\
\hline \multicolumn{4}{|l|}{ Non-essential amino acid } \\
\hline 1-methylhistidine & - & - & -0.651 \\
\hline Alanine & 0.750 & 0.778 & - \\
\hline Betaine & 0.812 & 0.834 & -0.769 \\
\hline Creatine & 0.930 & 0.826 & -0.849 \\
\hline Glycine & 0.871 & 0.674 & - \\
\hline Histidine & 0.776 & 0.602 & - \\
\hline Tyrosine & 0.832 & 0.859 & - \\
\hline
\end{tabular}

Table 2 OPLS-DA coefficients of metabolites in different pair-comparisons derived from NMR-data (Continued)

\begin{tabular}{|c|c|c|c|}
\hline \multirow[t]{2}{*}{ Metabolites } & \multicolumn{3}{|l|}{$r^{a}$} \\
\hline & $\begin{array}{l}\text { BxPC-3 vs } \\
\text { SW1990 }\end{array}$ & $\begin{array}{l}\text { Panc-1 vs } \\
\text { SW1990 }\end{array}$ & $\begin{array}{l}\text { Panc-1 vs } \\
\text { BxPC-3 }\end{array}$ \\
\hline \multicolumn{4}{|l|}{ Essential amino acid } \\
\hline Isoleucine & 0.749 & 0.795 & - \\
\hline Leucine & 0.707 & 0.775 & - \\
\hline Lysine & 0.886 & 0.822 & -0.780 \\
\hline Methionine & - & 0.645 & - \\
\hline Phenylalanine & 0.878 & 0.813 & -0.642 \\
\hline Threonine & 0.630 & 0.794 & 0.730 \\
\hline Tryptophan & 0.846 & 0.847 & -0.673 \\
\hline Valine & 0.839 & 0.858 & - \\
\hline \multicolumn{4}{|l|}{ Others } \\
\hline Methylguanidine & 0.650 & 0.732 & - \\
\hline Allantoin & -0.687 & - & - \\
\hline $\mathrm{N}$-acetyl glycoprotein & - & 0.661 & 0.914 \\
\hline Trimethylamine & -0.855 & 0.750 & 0.782 \\
\hline
\end{tabular}

serve as programmed switch for cell differentiation [53]. For instance, several NEAAs including glycine could be associated with gene signatures of cell proliferation and $M y c$ target activation through the serine-glycine-onecarbon pathway (SGOC pathway), which contribute significantly to energy generation and biosynthesis of $\mathrm{NADPH}$ and purine [54]. In addition, the mTORdependent induction of SGOC pathways can also lead to DNA methylation and tumorigenesis under the cooperatively oncogenic function of the loss of liver kinase B1 and activation of Kras, which highly involved in epigenetics [55]. Thus, NEAAs are highly associated with genesis, progression and epigenetics, and their relative concentration in serum may be indicators for the differentiation of PDAC.

\section{Relative high concentration metabolites of choline metabolism may imply a poor differentiation} Impressively, the high correlation coefficient of choline groups in the pair-comparison of Panc-1 vs BxPC-3 and Panc-1 vs SW1990 implied that relatively high concentration of choline-like metabolites including phosphocholine (PC) and glycerolphosphocholine (GPC) may be significant metabolic features for poor differentiation of PDAC. According to previous study, the tumor-associated choline metabolism plays a key role in cell malignant transformation, tumor migration and metastasis [56, 57], characterized by elevated level of PC and total choline in tissue $[45,46]$. Thereinto, the 


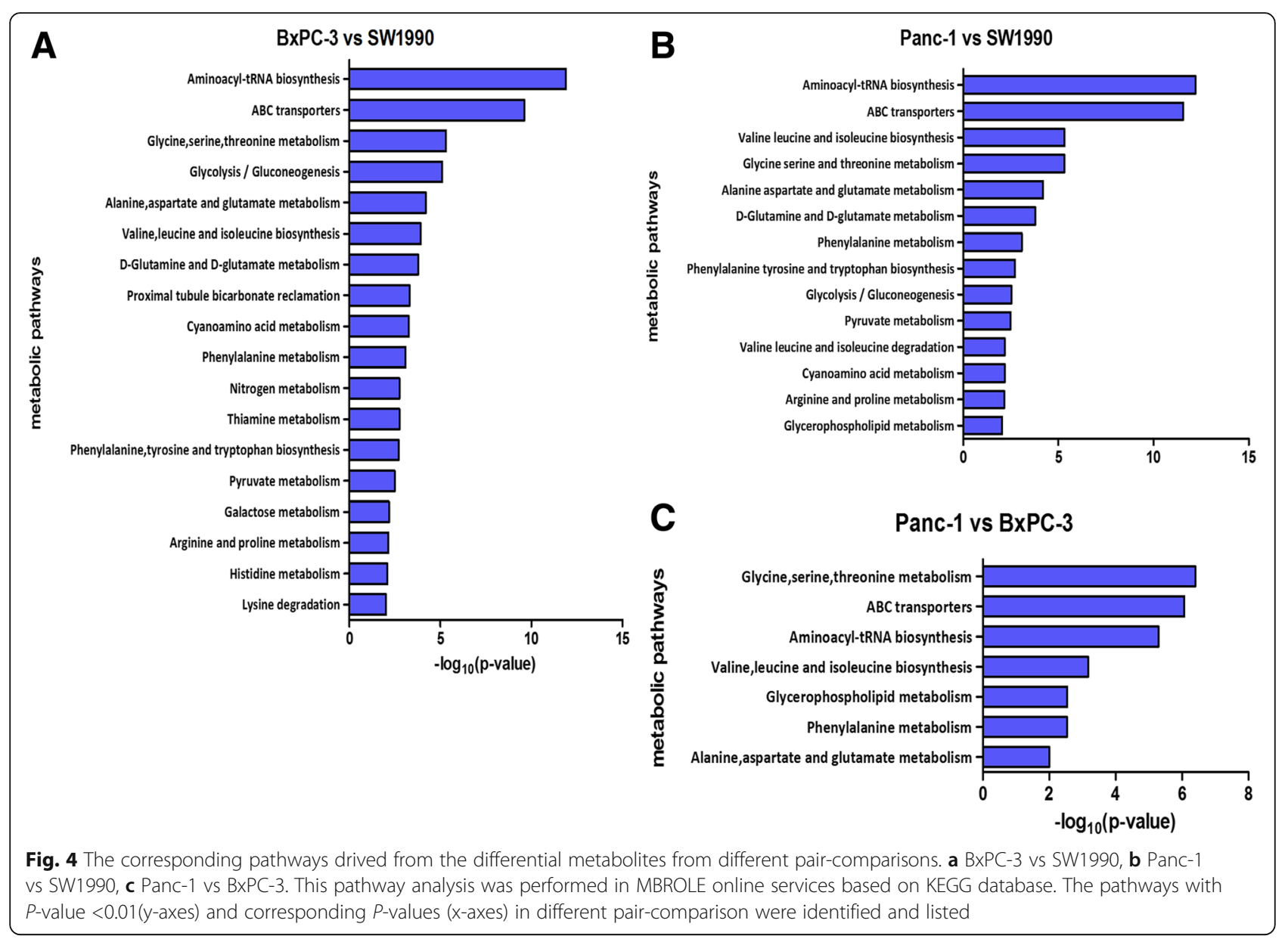

overexpression of choline kinase- $\alpha$ (Chk-a) induced by hypoxia-inducible factor (HIF) accounts for the increase of cellular PC and total choline [58], generating excessive phosphatidylcholine for biosynthesis of cell membrane. In addition, the EDI3-intermediated choline metabolism, a pathways verified in other solid tumor, can not only cleave GPC to form choline to supplement Kennedy pathway, but also generate glycerol-3-phosphate and its sequentially downstream intermediators for cellular signaling to regulate migration, invasion, proliferation and differentiation [57]. Other research detecting serum from PDAC patients also indicated that the choline metabolism were obviously altered and could potentially serve as biomarkers to detect PDAC in early stage. Thus, the difference of choline metabolism in PDAC could reflect and create a handful of regulatory functions on tumor progression and differentiation.

There are some pitfalls in this study. The PDAC models were established by using three PDAC cell lines which could only represent a part of metabonome landscape of pancreatic cancer, which inevitably lower the level of evidence provided from our study. The heterogeneity of pancreatic cancer in patients may compromise the directly clinical transformation application of our results. Thus, the further validation based on a large patient cohort will be performed in the future.

\section{Conclusions}

In this study, we compared the serum metabonomic profiling between PDAC with different differentiations and successfully established pattern recognition models to distinguish with each other. The lower concentration of amino acids, glycolytic and glutaminolytic participators may serve as the predictors for poor differentiation of tumor. Thus, NMR-based metabonomic strategy can be a promising non-invasive approach to predict tumor differentiation preoperatively.

\footnotetext{
Abbreviations

1-MH: 1-Methylhistidine; 3-HB: 3-Hydroxybutyrate; AA: Acetoacetate; Ace: Acetate; Act: Acetone; Ala: Alanine; All: Allantoin; Bet: Betaine;

Cho: Choline; Cit: Citrate; CPMG: Carr-Purcell-Meiboom-Gill; Cr: Creatine; EAAs: Essential amino acids; Eth: Ethanol; For: Formate; Fum: Fumarate; G: Glycerol; Gln: Glutamine; Glu: Glutamate; Gly: Glycine;

GPC: Glycerolphosphocholine; His: Histidine; HOD: Residual water signal; IB: Isobutyrate; Ile: Isoleucine; L1: LDL; L2: VLDL; Lac: Lactate; LAT-1: L-type amino-acid transporter 1; Leu: Leucine; Lys: Lysine; Mal: Malonate; Met: Methionine; MG: Methylguanidine; m-I: myo-Inositol; Mol: Methanol; mTOR: Mammalian target-of-rapamycin; NAG: N-acetyl glycoprotein;
} 
NEAAs: Non-essential amino acids; NMR: Nuclear magnetic resonance; OPLSDA: Orthogonal partial least squares discriminant analysis;

PC: Phosphocholine; PCA: Principal component analysis; PDAC: Pancreatic ductal adenocarcinoma; Phe: Phenylalanine; PLS-DA: Partial least squares discriminant analysis; Py: Pyruvate; SGOC pathway: Serine-glycine-one-carbon pathway; Suc: Succinate; TCA: Tricarboxylic acid; Thr: Threonine; TMA: Trimethylamine; Trp: Tryptophan; Tyr: Tyrosine; Val: Valine; a-Glc: a-Glucose; $\beta$-Glc: $\beta$-Glucose

\section{Acknowledgements}

No applicable

\section{Funding}

This work is sponsored by a. the National Natural Science Foundation of China (No. 81272581, 31671920); b. the United Fujian Provincial Health and Education Project for Tackling the Key Research (No.WKJ-FJ-10); c. National Key Clinical Specialty Discipline Construction Program of China and Key Clinical Specialty Discipline Construction Program of Fujian.

\section{Availability of data and materials}

The datasets generated and analyzed during the current study are not publicly available due to no available and appropriate public database can be achieved, but are available from the corresponding author on reasonable request.

\section{Authors' contributions}

SW established the animal models, interpreted the metabonomic data regarding PDAC and was the main writer for this manuscript. BZ was in charge of NMR detection of samples and the disposition of spectrum. JF designed the protocol of NMR detection, check the whole experimental data and was a major contributor in writing the manuscript. $\mathrm{WH}, \mathrm{XL}$ and JB were major contributors to animal models and the preparation of samples. $\mathrm{HH}$ was the initiator of this study, design the whole experimental protocol and as the ultimate checker for this manuscript. All authors have read and approved the final version of this manuscript.

\section{Ethics approval}

This experimental protocol was in accordance with the principles of National Institutes of Health guide for the care and use of laboratory animals and approved by Ethical Committee of Fujian Medical University.

\section{Consent for publication}

No applicable

\section{Competing interests}

The authors declare that they have no competing interests.

\section{Publisher's Note}

Springer Nature remains neutral with regard to jurisdictional claims in published maps and institutional affiliations.

\section{Received: 3 December 2016 Accepted: 25 October 2017}

Published online: 02 November 2017

\section{References}

1. Siegel RL, Miller KD, Jemal A. Cancer statistics, 2015. CA Cancer J Clin. 2015; 65(1):5-29.

2. Sohn T, Yeo C, Lillemoe K, Koniaris L, Kaushal S, Sauter P, Coleman J, Hruban R, Cameron J. Resected adenocarcinoma of the pancreas - 616 patients: results, outcome and prognostic indicators. Gastroenterology. 2000;118(4):A1059.

3. Winter J, Cameron J, Campbell K, Chang D, Coleman J, Hodgin M, Sauter P, Hruban R, Riall T, Schulick R. 1423 Pancreaticoduodenectomies for pancreatic cancer: a single-institution experience. J Gastrointest Surg. 2006; 10(9):1199-211.

4. Makowiec F, Riediger H, Fischer E, Keck T, Opitz OG, Adam U, Hopt UT. M1547 the lymph node-ratio is the strongest factor predicting survival after resection of pancreatic cancer. Gastroenterology. 2008;134(4):A-870

5. Nagai S, Fujii T, Kodera Y, Kanda M, Sahin TT, Kanzaki A, Yamada S, Sugimoto H, Nomoto S, Takeda S. Impact of operative blood loss on survival in invasive Ductal Adenocarcinoma of the pancreas. Pancreas. 2010:40(1):3-9.
6. Shimada K, Sano T, Sakamoto Y, Kosuge T. Clinical implications of combined portal vein resection as a palliative procedure in patients undergoing pancreaticoduodenectomy for pancreatic head carcinoma. Ann Surg Oncol. 2006:13(12):1569-78

7. Mitsuro K, Tsutomu F, Sahin TT, Akiyuki K, Shunji N, Suguru Y, Hiroyuki S, Shuji N, Shin T, Yasuhiro K. Invasion of the splenic artery is a crucial prognostic factor in carcinoma of the body and tail of the pancreas. Ann Surg. 2010;251(3):483-7.

8. Sugiura T, Uesaka K, Mihara K, Sasaki K, Kanemoto H, Mizuno T, Okamura Y. Margin status, recurrence pattern, and prognosis after resection of pancreatic cancer. Surgery. 2013;154(5):1078-86.

9. Neoptolemos JP, Stocken DD, Dunn JA, Almond J, Beger HG, Pederzoli P, Bassi C, Dervenis C, Fernandez-Cruz L, Lacaine F, et al. Influence of resection margins on survival for patients with pancreatic cancer treated by adjuvant chemoradiation and/or chemotherapy in the ESPAC-1 randomized controlled trial. Ann Surg. 2001;234(6):758-68.

10. Saukkonen K, Hagstrom J, Mustonen H, Juuti A, Nordling S, Kallio P, Alitalo K, Seppanen H, Haglund C. PROX1 and beta-catenin are prognostic markers in pancreatic ductal adenocarcinoma. BMC Cancer. 2016;16:472.

11. Reiser-Erkan C, Erkan M, Pan Z, Bekasi S, Giese NA, Streit S, Michalski CW, Friess $\mathrm{H}$, Kleeff J. Hypoxia-inducible proto-oncogene Pim-1 is a prognostic marker in pancreatic ductal adenocarcinoma. Cancer Biol Ther. 2008;7(9):1352-9.

12. Neoptolemos JP, Stocken DD, Friess H, Bassi C, Dunn JA, Hickey H, Beger $H$, Fernandez-Cruz L, Dervenis C, Lacaine F, et al. A randomized trial of chemoradiotherapy and chemotherapy after resection of pancreatic cancer. N Engl J Med. 2004;350(12):1200-10.

13. Barugola G, Partelli S, Marcucci S, Sartori N, Capelli P, Bassi C, Pederzoli P, Falconi M. Resectable pancreatic cancer: who really benefits from resection? Ann Surg Oncol. 2009;16(12):3316-22.

14. Hsu CC, Wolfgang CL, Laheru DA, Pawlik TM, Swartz MJ, Winter JM, Robinson R, Edil BH, Narang AK, Choti MA, et al. Early mortality risk score: identification of poor outcomes following upfront surgery for resectable pancreatic cancer. J Gastrointest Surg. 2012;16(4):753-61.

15. Joliat GR, Petermann D, Demartines N, Schafer M. External assessment of the early mortality risk score in patients with adenocarcinoma undergoing pancreaticoduodenectomy. HPB (Oxford). 2015;17(7):605-10.

16. Mornex F, Girard N, Delpero JR, Partensky C. Radiochemotherapy in the management of pancreatic cancer-part I: neoadjuvant treatment. Semin Radiat Oncol. 2005;15(4):226-34.

17. Quiros RM, Brown KM, Hoffman JP. Neoadjuvant therapy in pancreatic cancer. Cancer Investig. 2007;25(4):267-73.

18. Varadhachary GR, Tamm EP, Abbruzzese $\mathrm{JL}$, Xiong HQ, Crane $\mathrm{CH}$, Wang $\mathrm{H}$, Lee JE, Pisters PW, Evans DB, Wolff RA. Borderline resectable pancreatic cancer: definitions, management, and role of preoperative therapy. Ann Surg Oncol. 2006;13(8):1035-46.

19. Larghi A, Correale L, Ricci R, Abdulkader I, Monges G, Iglesias-Garcia J, Giovannini M, Attili F, Vitale G, Hassan C, et al. Interobserver agreement and accuracy of preoperative endoscopic ultrasound-guided biopsy for histological grading of pancreatic cancer. Endoscopy. 2015:47(4):308-14.

20. Nurmi A, Haglund C, Mustonen $H$, Seppänen H. Neoadjuvant therapy offers longer survival for pancreatic cancer patients over upfront surgery. Pancreatology. 2017;17(3):S73.

21. Mokdad AA, Minter RM, Zhu H, Augustine MM, Porembka MR, Wang SC, Yopp AC, Mansour JC, Choti MA, Polanco PM. Neoadjuvant therapy followed by resection versus upfront resection for Resectable pancreatic cancer: a propensity score matched analysis. J Clin Oncol. 2016;35(4):515-22.

22. Network NCC. NCCN clinical practice guidelines in oncology: pancreatic Adenocarcinoma(version 2.2014). Fort Washington: National Comprehensive Cancer Network; 2014. http://www.nccn.org/professionals/physician_gls/ $\mathrm{pdf} /$ pancreatic.pdf

23. Farrell JM, Pang JC, Kim GE, Tabatabai ZL. Pancreatic neuroendocrine tumors: accurate grading with $\mathrm{Ki}-67$ index on fine-needle aspiration specimens using the WHO 2010/ENETS criteria. Cancer Cytopathol. 2014;122(10):770-8.

24. Pitman MB, Centeno BA, Genevay M, Fonseca R, Mino-Kenudson M. Grading epithelial atypia in endoscopic ultrasound-guided fine-needle aspiration of intraductal papillary mucinous neoplasms: an international interobserver concordance study. Cancer Cytopathol. 2013;121(12):729-36.

25. Brindle JT, Antti H, Holmes E, Tranter G, Nicholson JK, Bethell HWL, Clarke S, Schofield PM, Mckilligin E, Mosedale DE. Rapid and noninvasive diagnosis of the presence and severity of coronary heart disease using 1H-NMR-based metabonomics. Nat Med. 2002;8(12):1439-44. 
26. Bartella L, Morris EA, Dershaw DD, Liberman L, Thakur SB, Moskowitz C, Guido J, Huang W. Proton MR spectroscopy with choline peak as malignancy marker improves positive predictive value for breast cancer diagnosis: preliminary study. Radiology. 2006;239(3):686-92.

27. Huzjan R, Sala E, Hricak H. Magnetic resonance imaging and magnetic resonance spectroscopic imaging of prostate cancer. Nat Clin Pract Urol. 2005;2(9):434-42.

28. Khan SA, Cox IJ, Thillainayagam AV, Bansi DS, Thomas HC, TaylorRobinson SD. Proton and phosphorus-31 nuclear magnetic resonance spectroscopy of human bile in hepatopancreaticobiliary cancer. Eur J Gastroenterol Hepatol. 2005;17(7):733-8.

29. Kwock L, Smith JK, Castillo M, Ewend MG, Collichio F, Morris DE, Bouldin TW, Cush S. Clinical role of proton magnetic resonance spectroscopy in oncology: brain, breast, and prostate cancer. Lancet Oncol. 2006;7(10):859-68.

30. Rehman L, Rehman UL, Azmat SK, Mohammad Hashim AS. Magnetic resonance spectroscopy: novel non-invasive technique for diagnosing brain tumors. J Coll Physicians Surg Pak. 2015;25(12):863-6.

31. Thomas MA, Wyckoff N, Yue K, Binesh N, Banakar S, Chung HK, Sayre J, DeBruhl N. Two-dimensional MR spectroscopic characterization of breast cancer in vivo. Technol Cancer Res Treat. 2005;4(1):99-106.

32. Kaplan O, Kushnir T, Askenazy N, Knubovets T, Navon G. Role of nuclear magnetic resonance spectroscopy (MRS) in cancer diagnosis and treatment: 31P, 23Na, and $1 \mathrm{H}$ MRS studies of three models of pancreatic cancer. Cancer Res. 1997;57(8):1452-9.

33. Bathe OF, Shaykhutdinov R, Kopciuk K, Weljie AM, McKay A, Sutherland FR, Dixon E, Dunse N, Sotiropoulos D, Vogel HJ. Feasibility of identifying pancreatic cancer based on serum metabolomics. Cancer Epidemiol Biomarkers Prev. 2011;20(1):140-7.

34. Zhang L, Jin H, Guo X, Yang Z, Zhao L, Tang S, Mo P, Wu K, Nie Y, Pan Y, et al. Distinguishing pancreatic cancer from chronic pancreatitis and healthy individuals by $(1) \mathrm{H}$ nuclear magnetic resonance-based metabonomic profiles. Clin Biochem. 2012;45(13-14):1064-9.

35. Fang $F$, He X, Deng H, Chen Q, Lu J, Spraul M, Yu Y. Discrimination of metabolic profiles of pancreatic cancer from chronic pancreatitis by high-resolution magic angle spinning $1 \mathrm{H}$ nuclear magnetic resonance and principal components analysis. Cancer Sci. 2007;98(11):1678-82.

36. Tan MH, Nowak NJ, Loor R, ., Ochi H, ., Sandberg AA, Lopez C, ., Pickren JW, Berjian $\mathrm{R}$.., Douglass HO, Chu TM: Characterization of a new primary human pancreatic tumor line. Cancer Investig 1986, 4(1):15-23.

37. Kyriazis AP, Sandberg AA, Kyriazis AA, Sloane NH, Lepera R. Establishment and characterization of human pancreatic adenocarcinoma cell line SW-1990 in tissue culture and the nude mouse. Cancer Res. 1983:43(9):4393-401.

38. Lieber M, ., Mazzetta J, ., Nelson-Rees W, ., Kaplan M, ., Todaro G,. Establishment of a continuous tumor-cell line (panc-1) from a human carcinoma of the exocrine pancreas. Int J Cancer 1975, 15(5):741-747.

39. Deer EL, Jessica GH, Coursen JD, Shea JE, Josephat N, Scaife CL, Firpo MA, Mulvihill SJ. Phenotype and genotype of pancreatic cancer cell lines. Pancreas. 2010;39(4):425-35

40. Chagoyen M, Pazos F. MBRole: enrichment analysis of metabolomic data. Bioinformatics. 2011;27(5):730-1.

41. Kanehisa M, Goto S, Sato Y, Kawashima M, Furumichi M, Tanabe M. Data, information, knowledge and principle: back to metabolism in KEGG. Nucleic Acids Res. 2014;42(Database issue):D199-205.

42. Wishart DS, Knox C, Guo AC, Eisner R, Young N, Gautam B, Hau DD, Psychogios N, Dong E, Bouatra S, et al. HMDB: a knowledgebase for the human metabolome. Nucleic Acids Res. 2009;37(Database issue): D603-10.

43. Cui Q, Lewis IA, Hegeman AD, Anderson ME, Li J, Schulte CF, Westler WM, Eghbalnia HR, Sussman MR, Markley JL. Metabolite identification via the Madison Metabolomics consortium database. Nat Biotechnol. 2008;26(2):162-4.

44. Hirschhaeuser F, Sattler UG, Mueller-Klieser W. Lactate: a metabolic key player in cancer. Cancer Res. 2011;71(22):6921-5.

45. Guillaumond F, Leca J, Olivares O, Lavaut MN, Vidal N, Berthezene P, Dusetti NJ, Loncle C, Calvo E, Turrini O, et al. Strengthened glycolysis under hypoxia supports tumor symbiosis and hexosamine biosynthesis in pancreatic adenocarcinoma. Proc Natl Acad Sci U S A. 2013;110(10):3919-24.

46. OuYang D, Xu J, Huang $H$, Chen Z. Metabolomic profiling of serum from human pancreatic cancer patients using 1H NMR spectroscopy and principal component analysis. Appl Biochem Biotechnol. 2011;165(1):148-54.
47. Kobayashi T, Nishiumi S, Ikeda A, Yoshie T, Sakai A, Matsubara A, Izumi Y, Tsumura H, Tsuda M, Nishisaki H, et al. A novel serum metabolomics-based diagnostic approach to pancreatic cancer. Cancer Epidemiol Biomarkers Prev. 2013;22(4):571-9.

48. Soga T. Cancer metabolism: key players in metabolic reprogramming. Cancer Sci. 2013;104(3):275-81.

49. Son J, Lyssiotis CA, Ying H, Wang X, Hua S, Ligorio M, Perera RM, Ferrone CR, Mullarky E, Shyh-Chang N, et al. Glutamine supports pancreatic cancer growth through a KRAS-regulated metabolic pathway. Nature. 2013; 496(7443):101-5.

50. Mayers JR, Wu C, Clish CB, Kraft P, Torrence ME, Fiske BP, Yuan C, Bao Y, Townsend MK, Tworoger SS, et al. Elevation of circulating branched-chain amino acids is an early event in human pancreatic adenocarcinoma development. Nat Med. 2014;20(10):1193-8.

51. Fuchs BC, Bode BP. Amino acid transporters ASCT2 and LAT1 in cancer: partners in crime? Semin Cancer Biol. 2005;15(4):254-66.

52. Kaira K, Sunose Y, Arakawa K, Ogawa T, Sunaga N, Shimizu K, Tominaga H, Oriuchi N, Itoh H, Nagamori S, et al. Prognostic significance of L-type amino-acid transporter 1 expression in surgically resected pancreatic cancer. Br J Cancer. 2012;107(4):632-8.

53. Phang JM, Liu W, Hancock C. Bridging epigenetics and metabolism: role of non-essential amino acids. Epigenetics. 2013;8(3):231-6.

54. Tedeschi PM, Markert EK, Gounder M, Lin H, Dvorzhinski D, Dolfi SC, Chan LL, Qiu J, DiPaola RS, Hirshfield KM, et al. Contribution of serine, folate and glycine metabolism to the ATP, NADPH and purine requirements of cancer cells. Cell Death Dis. 2013:4:e877.

55. Kottakis F, Nicolay BN, Roumane A, Karnik R, Gu H, Nagle JM, Boukhali M, Hayward MC, Li YY, Chen T, et al. LKB1 loss links serine metabolism to DNA methylation and tumorigenesis. Nature. 2016;539(7629):390-95.

56. Glunde K, Bhujwalla ZM, Ronen SM. Choline metabolism in malignant transformation. Nat Rev Cancer. 2011;11(12):835-48.

57. Stewart JD, Marchan R, Lesjak MS, Lambert J, Hergenroeder R, Ellis JK, Lau $\mathrm{CH}$, Keun HC, Schmitz G, Schiller J, et al. Choline-releasing glycerophosphodiesterase EDI3 drives tumor cell migration and metastasis. Proc Natl Acad Sci U S A. 2012;109(21):8155-60.

58. Penet MF, Shah T, Bharti S, Krishnamachary B, Artemov D, Mironchik Y, Wildes F, Maitra A, Bhujwalla ZM. Metabolic imaging of pancreatic ductal adenocarcinoma detects altered choline metabolism. Clin Cancer Res. 2015; 21(2):386-95.

\section{Submit your next manuscript to BioMed Central and we will help you at every step:}

- We accept pre-submission inquiries

- Our selector tool helps you to find the most relevant journal

- We provide round the clock customer support

- Convenient online submission

- Thorough peer review

- Inclusion in PubMed and all major indexing services

- Maximum visibility for your research

Submit your manuscript at www.biomedcentral.com/submit 